\title{
O trabalho da enfermagem no pós-operatório de cirurgia cardíaca: uma revisão integrativa
}

\author{
The work of nursing in the postoperative period of cardiac surgery: \\ an integrative review
}

Ana Paula Azevedo Santos ${ }^{1}$, Ana Maria Laus ${ }^{1}$, Silvia Helena Henriques Camelo

${ }^{1}$ Escola de Enfermagem de Ribeirão Preto da Universidade de São Paulo (USP) - Ribeirão Preto (SP), Brasil.

DOI: http://dx.doi.org/10.7322/abcshs.v40i1.703

\section{RESUMO}

Este estudo teve o objetivo de identificar, por meio de produções científicas nacionais e internacionais, os cuidados desenvolvidos pela equipe de enfermagem durante a assistência ao paciente no pósoperatório de cirurgia cardíaca. Trata-se de uma revisão integrativa realizada por meio de busca às bases PubMed, LILACS, CINAHL, SCOPUS e BDENF no período de fevereiro a junho de 2014, com o seguinte questionamento: "Quais os cuidados realizados pela equipe de enfermagem ao paciente no pós-operatório de cirurgia cardíaca?" Utilizou-se como critério de inclusão artigos científicos publicados nos últimos cinco anos, em português, inglês e espanhol, disponíveis na íntegra. Foram selecionados 21 artigos e, mediante a sua leitura criteriosa, foi realizada análise descritiva, possibilitando o agrupamento dos dados em um núcleo temático: cuidando do paciente no pós-operatório de cirurgia cardíaca, que apontou subcategorias: assistindo o paciente após cirurgia cardíaca, gerenciando a dor e a comunicação como ferramenta no cuidado de enfermagem. Os cuidados desenvolvidos no pós-operatório de cirurgia cardíaca demonstram a necessidade de organização do processo de trabalho da equipe de enfermagem bem como competências específicas dos profissionais para atuarem nesse cenário, com intuito de promover um cuidado individualizado e qualificado.

Palavras-chave: cuidados de enfermagem; cirurgia torácica; período pós-operatório.

\section{ABSTRACT}

This study aimed to identify, through national and international scientific production, the care provided by the nursing staff during patient care in the postoperative period of cardiac surgery. It is an integrative review accomplished through searching PubMed, LILACS, CINAHL, SCOPUS and BDENF in the period from February to June 2014, with the question: "What is the care provided to the patient by nursing staff in the postcardiac surgery?" It was used as a criterion for inclusion scientific articles published in the last five years, in Portuguese, English and Spanish, available in its entirety. Twenty-one articles were selected and, by careful reading, descriptive analysis was performed, enabling the pooling of data on a theme: caring for the patient during the postoperative period of cardiac surgery, which yielded subcategories: taking care of the patient in the postoperative period of cardiac surgery, managing pain and communication as a tool in nursing care. The care in the postoperative period of cardiac surgery demonstrate the necessity of organization of the work process of the nursing staff as well as specific professional skills to work in this scenario, in order to promote individualized and qualified care.

Keywords: nursing care; thoracic surgery; postoperative period. 


\section{INTRODUÇÃO}

No cenário das doenças crônicas não transmissíveis (DCNT), as doenças cardiovasculares são frequentes causas de morbimortalidade, apresentando como fator de risco envolvido na gênese das DCNT o crescente processo de envelhecimento da população ${ }^{1}$, que associado ao acesso inadequado aos serviços de saúde de alta qualidade, incluindo deficiências na prevenção, nos serviços de diagnóstico e o difícil acesso aos medicamentos essenciais, faz das DCNT relevantes no contexto mundial, principalmente nos países em desenvolvimento ${ }^{2}$.

No Brasil, esse grupo de doenças é responsável por $72 \%$ da mortalidade ${ }^{3}$. A doença cardíaca isquêmica e o acidente vascular cerebral são as duas principais causas de mortalidade em todo mundo, correspondendo, respectivamente, a 13,2 e 11,9\% do total de óbitos em $2012^{4}$. No Brasil, a situação é mais alarmente, uma vez que 31,9\% dos óbitos ocorridos em 2007 tiveram como causa as doenças do aparelho circulatório ${ }^{5}$. No Estado de São Paulo, as doenças do aparelho circulatório representaram a principal causa de mortalidade geral entre os anos de 1997 e $2007^{6}$.

Particularmente, no tocante à atenção cardiovascular, a Portaria $\mathrm{n}^{\circ}$ 1.1169/GM, de 15 de junho de 2004, institui a Política Nacional de Atenção Cardiovascular de Alta Complexidade, que tem como um dos seus princípios atender integralmente aos portadores de patologias cardiovasculares no Sistema Único de Saúde (SUS), organizar a assistência em serviços hierarquizados e regionalizados e garantir a assistência nos vários níveis de complexidade ${ }^{7}$.

O tratamento das doenças cardíacas pode ser clínico ou cirúrgico $^{8-10}$. O desenvolvimento tecnológico na área de Medicina tem permitido que operações cardiovasculares fossem conduzidas com segurança e resultados melhores. Fatores institucionais e pessoais estão envolvidos nesses bons resultados para melhor atendimento na assistência pós-operatória, exigindo dos profissionais atitudes, conhecimentos e habilidades específicas ${ }^{11,12}$.

No campo de atuação do enfermeiro, as doenças cardíacas correspondem a importante demanda de cuidados, justificando um olhar sistematizado para esse grupo de agravos, na perspectiva da integralidade da atenção. Nesse âmbito, o paciente submetido à cirurgia cardíaca exige cuidados de enfermagem fundamentados nas necessidades técnico-científicas, cirúrgicas, emocionais e psicossociais, as quais devem ser observadas e respeitadas, viabilizando a qualidade do processo pós-operatório ${ }^{13}$.

A unidade pós-operatória de cirurgia cardíaca é caracterizada como uma unidade de alta complexidade, tendo como objetivo monitorar a recuperação e possíveis complicações decorrentes do ato cirúrgico ${ }^{14,15}$. A complexidade e pormenores dos cuidados requeridos por pacientes submetidos a cirurgias cárdicas, cuja situação de saúde sofre mudanças constantes e abruptas, requerem observação contínua e intervenções de enfermagem imediatas e precisas, fazendo-se necessários a organização e planejamento prévio e científico ${ }^{16}$.

O cenário de inovação e atendimento especializado realizado pelo enfermeiro leva à necessidade do desenvolvimento de uma metodologia própria de trabalho, sendo fundamentada no processo de enfermagem e exigindo do enfermeiro o desenvolvimento de competências ${ }^{17}$.

Considerando a nossa experiência profissional enquanto enfermeiras e pesquisadoras, conhecendo e atuando em unidades pós-operatórias que prestam atendimento a pacientes submetidos a cirurgias cardíacas, temos observado elevada demanda pelos cuidados de enfermagem com diferentes graus de exigências, fazendo-se necessário aprofundar o conhecimento a respeito deles, pois os mesmos podem ser potencialmente comprometedores do estado de saúde do paciente.

Assim, este estudo apresenta o seguinte questionamento: quais os cuidados realizados pela equipe de enfermagem ao paciente no pós-operatório de cirurgia cardíaca?

Identificar os cuidados realizados pela equipe de enfermagem deve provocar reflexão desses profissionais, dos centros formadores e gestores sobre a complexidade do trabalho desenvolvido pela equipe de enfermagem na assistência ao paciente cardíaco, apontando ferramentas e/ou estratégias para um atendimento de qualidade.

Nesse sentido, propôs-se esta investigação, com o objetivo de identificar, por meio de produções científicas nacionais e internacionais, os cuidados desenvolvidos pela equipe de enfermagem durante a assistência ao paciente no pós-operatório de cirurgia cardíaca.

\section{MÉTODO}

Para o alcance do nosso objetivo, optamos pelo método da revisão integrativa da literatura, visto que é a mais ampla abordagem metodológica referente às revisões e possibilita a síntese e análise do conhecimento científico já produzido sobre o tema investigado ${ }^{18}$.

A seleção dos artigos foi realizada no período de fevereiro a junho de 2014 por meio de consulta às seguintes bases de dados: PubMed (Literatura Biomédica norte-americana, criado pela US Nacional Library of Medicine), LILACS (Literatura Latino-Americana e do Caribe em Ciências da Saúde), CINAHL (Cumulative Index to Nursing and Allied Health Literature), SCOPUS e BDENF (Base de Dados Bibliográficos Especializada na Área de Enfermagem do Brasil).

Os Descritores em Ciências da Saúde (DeCS) utilizados para a busca foram: "cuidados de enfermagem" ("nursing care"), "cirurgia torácica" ("thoracic surgery") e "período pós-operatório" ("postoperative period"). Procurou-se ampliar o âmbito da pesquisa, reduzindo possíveis limitações decorrentes do uso de descritores, realizando a busca, também, utilizando os sinônimos dos descritores e os descritores como palavras-chave. Além disso, em virtude das características específicas de cada base de dados, as estratégias utilizadas para localizar os artigos foram adaptadas para cada uma, tendo como eixo a pergunta norteadora e os critérios de inclusão e exclusão, previamente estabelecidos para manter a coerência na busca dos artigos.

Os critérios de inclusão foram: artigos científicos publicados nos últimos cinco anos, em português, inglês e espanhol, 
disponíveis na íntegra e que abordassem a temática do estudo. Foram excluídos teses, dissertações, livros e capítulos de livros, de modo a selecionar apenas publicações em periódicos indexados.

Assim, mediante a leitura criteriosa dos artigos, a fim de verificar a adequação com a questão norteadora, selecionaram-se 13 artigos na PubMed, oito na LILACS, três no CINAHL, quatro no SCOPUS e quatro na BDENF, alguns encontrados em mais de uma base de dados, totalizando 21 publicações.

Os estudos selecionados foram classificados segundo o nível de evidência ${ }^{19} \mathrm{e}$ a análise foi realizada de forma descritiva, o que possibilitou o agrupamento dos dados em um núcleo temático: cuidando do paciente no pós-operatório de cirurgia cardíaca, apontando subcategorias: assistindo o paciente após cirurgia cardíaca, gerenciando a dor e a comunicação como ferramenta no cuidado de enfermagem.

\section{RESULTADOS E DISCUSSÃO}

\section{Caracterização dos artigos}

Foram selecionados 21 artigos, sendo a maioria de autoria de enfermeiros e 12 na língua portuguesa, conforme Quadro 1.

Ao analisarmos os delineamentos de pesquisa dos estudos, identificamos que 13 artigos utilizaram abordagem metodológica quantitativa, sete qualitativa e um quantitativo-qualitativo. Entre os estudos que utilizaram abordagem quantitativa, os métodos usados foram: ensaio clínico randomizado controlado, ensaio clínico bem delineado sem randomização, descritivo, transversal e exploratório. Os estudos qualitativos foram: estudo de caso, exploratório, descritivo, fenomenológico e documental. E na abordagem quantitativa-qualitativa, o método foi descritivo-exploratório.

Assim, os desenhos metodológicos dos artigos selecionados apontam níveis de evidência científica entre dois e seis, sendo nove estudos com nível de evidência variando entre dois e quatro e 12 estudos com nível de evidência seis. Portanto, os estudos ora avaliados contribuem para fortes evidências sobre a temática estudada.

\section{Cuidando do paciente no pós-operatório de cirurgia cardíaca}

\section{Assistindo o paciente após cirurgia cardíaca}

A tarefa de cuidar de pacientes após cirurgia cardíaca é uma atividade distribuída entre todos os membros da equipe de saúde, porém a equipe de enfermagem, por representar um contingente expressivo nesse contexto, merece atenção. As atividades desenvolvidas por essa equipe vão desde a coleta de informações sobre o paciente que ainda permanece na sala de cirurgia, o preparo da unidade de recuperação para admissão desse paciente até a assistência propriamente dita.

$\mathrm{Na}$ admissão do paciente na unidade pós-operatória de cirurgia cardíaca, os procedimentos e o monitoramento minucioso levam o enfermeiro a colocar em prática o seu conhecimento técnico-científico. O enfermeiro organiza a unidade e dimensiona a equipe de enfermagem, ações estas que aprimoram o desempenho na admissão e propiciam estrutura adequada para que a admissão aconteça com segurança, pois mesmo que a cirurgia tenha ocorrido com sucesso, o cuidado pós-operatório é determinante para o prognóstico ${ }^{20}$.

Além disso, as prioridades do paciente poderão variar de acordo com o período do pós-operatório, ou seja, imediato, mediato ou tardio. $\mathrm{O}$ cuidado nesses cenários contemplam as necessidades de cuidados específicos e indispensáveis diante da complexidade que envolve o paciente ${ }^{17}$.

Enfermeiros que atuam nesse cenário identificam como cuidados de enfermagem aqueles referentes à manutenção do débito cardíaco, da integridade tecidual, do equilíbrio hidroeletrolítico e da oxigenação. Para cada um desses itens, temos cuidados específicos, tais como: monitorização cardíaca; balanço hídrico; administração de hemoderivados; mudanças de decúbito; uso de curativos protetores; avaliar as condições da pele; observar necessidade de reposição hídrica; coletar e avaliar exames laboratoriais; oferecer oxigenioterapia conforme necessidade, e outros ${ }^{15}$.

Também descrevem cuidados relacionados à prevenção e controle de infecção, da dor e apoio psicológico, como: realizar lavagem das mãos, usar equipamentos de proteção individual, observar sinais de infecção em dispositivos invasivos, observar fácies de dor, administrar medicamentos, suporte religioso e outros ${ }^{15}$.

O paciente submetido à cirurgia cardíaca apresenta dependência da ventilação mecânica no período pós-operatório imediato, ou seja, em torno de 24 horas após a cirurgia, apresentando-se como o período mais crítico, exigindo maior observação da equipe multidisciplinar envolvida nos cuidados ${ }^{13,17}$. Um estudo retrata essa preocupação ao desenvolver e implementar um protocolo de prevenção de aspiração para pacientes após cirurgia cardíaca, demonstrando eficácia na redução de ocorrência de pneumonia na população do estudo ${ }^{21}$, o que vem ao encontro de outra investigação, onde se evidenciou a importância do cuidado de enfermagem referente a ventilação e oxigenação ${ }^{15}$.

Na equipe de enfermagem, o enfermeiro deve acompanhar os procedimentos essenciais, como banho no leito, de forma criteriosa e coerente ${ }^{17}$. O banho do paciente propicia à equipe de enfermagem avaliar a pele do mesmo e prescrever cuidados para a prevenção de feridas, como o uso de curativos profiláticos, que se mostraram eficientes na prevenção de úlceras por pressão em pacientes submetidos à cirurgia cardíaca ${ }^{22}$.

Outras intervenções foram citadas na literatura como fator para recuperação desses pacientes. Ouvir música aumenta os níveis de oxitocina e de relaxamento, proporcionando maior conforto durante a recuperação ${ }^{23}$. A massagem terapêutica como intervenção para melhorar psicometria, fisiologia e a ocorrência de fibrilação atrial em adultos submetidos à cirurgia cardíaca pode trazer resultados benéficos, dependendo da frequência e foco anatômico da massagem terapêutica ${ }^{24}$. 
Quadro 1: Apresentação dos artigos incluídos na revisão integrativa segundo ano de publicação, periódico, autores, resultados e nível de evidência. Ribeirão Preto, 2014.

\begin{tabular}{|c|c|c|c|c|}
\hline Ano & Periódico & Autores & Resultados & $\begin{array}{l}\text { Nível de } \\
\text { evidência }\end{array}$ \\
\hline 2013 & $\begin{array}{l}\text { Pain Manag } \\
\text { Nurs }\end{array}$ & $\begin{array}{l}\text { Özer N, Karaman Özlü Z, } \\
\text { Arslan S, Günes N }{ }^{35}\end{array}$ & $\begin{array}{l}\text { Uso da música levou a um aumento estatisticamente significativo da } \\
\text { saturação de oxigênio e menor escore de dor. }\end{array}$ & 3 \\
\hline 2013 & $\begin{array}{l}\text { Rev Esc } \\
\text { Enferm USP }\end{array}$ & $\begin{array}{l}\text { Keller C, Paixão A, } \\
\text { Moraes MA, Rabelo ER, } \\
\text { Goldmeier } \mathrm{S}^{31}\end{array}$ & $\begin{array}{l}\text { Capacitação no uso de escala de dor interferindo no tipo de } \\
\text { analgesia administrada em relação à intensidade da dor. }\end{array}$ & 3 \\
\hline 2012 & $\begin{array}{l}\text { Esc Anna } \\
\text { Nery }\end{array}$ & $\begin{array}{l}\text { Duarte SCM, Stipp MAC, } \\
\text { Mesquita MGR, Silva MM"17 }\end{array}$ & $\begin{array}{c}\text { O estudo mostrou enfermagem preocupada com o cuidado técnico à } \\
\text { beira do leito e que o processo de enfermagem contribuirá para uma } \\
\text { assistência de qualidade. }\end{array}$ & 6 \\
\hline 2012 & $\begin{array}{l}\text { Rev Min } \\
\text { Enferm }\end{array}$ & $\begin{array}{l}\text { Faria-Filho GS, Caixeta LR, } \\
\text { Stival MM, Lima LR }{ }^{28}\end{array}$ & $\begin{array}{l}\text { Esse estudo identificou a dor aguda do paciente por meio do } \\
\text { diagnóstico de enfermagem junto com instrumento unidimensional. }\end{array}$ & 4 \\
\hline 2012 & $\begin{array}{l}\text { Esc Anna } \\
\text { Nery }\end{array}$ & $\begin{array}{l}\text { Melo HC, Araújo SEG, } \\
\text { Santos VEFA, Veríssimo } \\
\text { AVR, Alves ERP, Souza } \\
\text { MHN } 20\end{array}$ & $\begin{array}{l}\text { Permitiu delinear o cuidado do enfermeiro como sistemático, } \\
\text { complexo e minucioso, que requer ao mesmo tempo aptidões } \\
\text { técnico-científicas. }\end{array}$ & 6 \\
\hline 2012 & $\begin{array}{l}\text { J Wound } \\
\text { Ostomy } \\
\text { Continence } \\
\text { Nurs }\end{array}$ & Brindle CT, Wegelin $\mathrm{JA}^{22}$ & $\begin{array}{l}\text { Cuidados de enfermagem como o uso de curativos profiláticos para } \\
\text { úlcera de pressão em pacientes submetidos a cirurgias cardíacas. }\end{array}$ & 2 \\
\hline 2012 & $\begin{array}{l}\text { Pain Manag } \\
\text { Nurs }\end{array}$ & $\begin{array}{l}\text { Bai J, Hsu L, Tang Y, van } \\
\text { Dijk } M^{32}\end{array}$ & $\begin{array}{c}\text { As escalas para avaliação da dor COMFORT-B e FLACC parecem } \\
\text { ser ferramentas úteis na avaliação da dor em crianças chinesas no } \\
\text { pós-operatório de cirurgia cardíaca. }\end{array}$ & 6 \\
\hline 2012 & $\begin{array}{l}\mathrm{J} \text { Med } \\
\text { Internet Res }\end{array}$ & $\begin{array}{l}\text { Martorella G, Côté J, } \\
\text { Racine M, Choinière } \mathrm{M}^{34}\end{array}$ & $\begin{array}{l}\text { A intervenção de enfermagem baseada na web pode aumentar a } \\
\text { acessibilidade à educação em saúde e promover o alívio da dor. }\end{array}$ & 2 \\
\hline 2012 & $\begin{array}{l}\text { Intensive Crit } \\
\text { Care Nurs }\end{array}$ & Connor $\mathrm{LO}^{33}$ & $\begin{array}{l}\text { Enfermeiros utilizaram diferentes combinações de sinais de dor } \\
\text { para fazer um julgamento do paciente ventilado evidenciado por } \\
\text { alterações fisiológicas e um "padrão de sugestão de comportamento } \\
\text { da dor" evidenciado por comportamentos. }\end{array}$ & 6 \\
\hline 2012 & $\begin{array}{l}\text { Rev Esc } \\
\text { Enferm USP }\end{array}$ & $\begin{array}{l}\text { Magalhães MGPA, Alves } \\
\text { LMO, Alcantara LFM, } \\
\text { Bezerra SMMS }\end{array}$ & $\begin{array}{c}\text { Observou-se alta letalidade por mediastinite, necessidade de } \\
\text { supervisão contínua da enfermagem e medidas preventivas para o } \\
\text { diagnóstico precoce. }\end{array}$ & 4 \\
\hline 2012 & Rev Rene & $\begin{array}{l}\text { Lira ALBC, Araújo WM, } \\
\text { Souza NTC, Frazão CMFQ, } \\
\text { Medeiros ABA }{ }^{15}\end{array}$ & $\begin{array}{l}\text { Identificaram-se cuidados de enfermagem agrupados em categorias: } \\
\text { manutenção do débito cardíaco, integridade tecidual, equilíbrio } \\
\text { hidroeletrolítico, ventilação e oxigenação, prevenção e tratamento da } \\
\text { dor, prevenção e controle da infecção e apoio psicológico. }\end{array}$ & 6 \\
\hline 2011 & $\begin{array}{l}\text { Crit Care } \\
\text { Nurse }\end{array}$ & Starks B, Harbert C ${ }^{21}$ & $\begin{array}{l}\text { Uso pela equipe de enfermagem de protocolo de prevenção de } \\
\text { aspiração em pacientes no pós- operatório de cirurgia cardíaca com } \\
\text { resultado positivo para evitar pneumonia. }\end{array}$ & 3 \\
\hline 2011 & $\begin{array}{l}\text { Ciênc Cuid } \\
\text { Saúde }\end{array}$ & $\begin{array}{l}\text { Gonçalves RMDA, Pereira } \\
\text { MER, Pedrosa LAK, Silva } \\
\text { QCG, Abreu RMD }\end{array}$ & $\begin{array}{l}\text { A comunicação entre o enfermeiro e o paciente como importante } \\
\text { veículo no perioperatório de cirurgia cardíaca. }\end{array}$ & 6 \\
\hline 2011 & $\begin{array}{l}\text { Rev Esc } \\
\text { Enferm USP }\end{array}$ & $\begin{array}{l}\text { Miranda AFA, Silva LF, } \\
\text { Caetano JA, Sousa AC, } \\
\text { Almeida PC } 29\end{array}$ & $\begin{array}{l}\text { A manifestação da dor ocorreu em diferentes classificações e a } \\
\text { principal alteração nos sinais vitais ocorreu na pressão arterial. }\end{array}$ & 6 \\
\hline 2010 & $\begin{array}{l}\text { Pain Manag } \\
\text { Nurs }\end{array}$ & Marmo L, Fowler $\mathrm{S}^{30}$ & $\begin{array}{l}\text { Escalas de avaliação da dor capturam adequadamente a dor no } \\
\text { paciente sedado em estado crítico não verbal com base na avaliação } \\
\text { da face dos paciente, dos movimentos corporais, tensão muscular, } \\
\text { respiração e sinais vitais. }\end{array}$ & 6 \\
\hline 2010 & $\begin{array}{l}\text { Rev } \\
\text { Latino-Am } \\
\text { Enfermagem }\end{array}$ & $\begin{array}{l}\text { Nascimento LC, Strabelli } \\
\text { BS, Almeida FCQG, } \\
\text { Rossato LM, Leite AM, Lima } \\
\text { RAG } \\
\text { RA }\end{array}$ & $\begin{array}{c}\text { Na avaliação de mães sobre o manejo da dor de seus filhos, cuidar } \\
\text { bem é resultante da confiança que elas depositam na equipe de } \\
\text { enfermagem e da observação das intervenções medicamentosas } \\
\text { que a equipe realiza. }\end{array}$ & 6 \\
\hline 2010 & $\begin{array}{l}\text { Acta Paul } \\
\text { Enferm }\end{array}$ & $\begin{array}{l}\text { Pivoto FL, Lunardi-Filho } \\
\text { WD, Santos SSC, Almeida } \\
\text { MA, Silveira RS }{ }^{16}\end{array}$ & $\begin{array}{c}\text { Foram estabelecidos diagnósticos de enfermagem no pós-operatório } \\
\text { de cirurgia cardíaca que permitem o direcionamento da assistência } \\
\text { de enfermagem. }\end{array}$ & 6 \\
\hline 2010 & Salusvita & Cruz APO, Lopes R ${ }^{27}$ & $\begin{array}{c}\text { A construção e validação de instrumentos de coleta de dados para } \\
\text { a identificação de diagnósticos de enfermagem no pós-operatório de } \\
\text { cirurgia cardíaca subsidiam a elaboração de um planejamento e a } \\
\text { melhoria dos cuidados de enfermagem. }\end{array}$ & 6 \\
\hline 2009 & Heart Lung & $\begin{array}{l}\text { Albert NM, Gillinov AM, } \\
\text { Lytle BW, Feng J, Cwynar } \\
\text { R, Blackstone } \mathrm{EH}^{24}\end{array}$ & $\begin{array}{c}\text { A massagem terapêutica pode ser viável em pacientes de } \\
\text { cirurgia cardíaca. }\end{array}$ & 2 \\
\hline 2009 & $\begin{array}{l}\text { Rev } \\
\text { SOCERJ }\end{array}$ & $\begin{array}{l}\text { Silva RCL, Kaczmarkiewicz } \\
\text { CC, Cunha JJSA, Meira IC, } \\
\text { Figueiredo NMA, Porto IS }{ }^{26}\end{array}$ & $\begin{array}{c}\text { Existem situações que podem contribuir com a desumanização na terapia } \\
\text { intensiva, como a falta de privacidade do cliente, supervalorização da } \\
\text { tecnologia e escassez de diálogo entre o profissional e o cliente. }\end{array}$ & 6 \\
\hline 2009 & J Clin Nurs & Nilsson $\mathrm{U}^{23}$ & $\begin{array}{l}\text { Uso da música aumenta os níveis de oxitocina levando ao aumento } \\
\text { dos níveis de relaxamento nos pacientes. }\end{array}$ & 2 \\
\hline
\end{tabular}


Outro aspecto que merece destaque na assistência a esses pacientes diz respeito ao risco de infecções. Uma forma de prevenir infecções por parte dos profissionais são ações simples como lavagem das mãos, uso adequado dos equipamentos de proteção individual e manutenção das técnicas assépticas, evitando-se reinternações desnecessárias ${ }^{25}$.

Nessa direção, assistir o paciente no pós-operatório de cirurgia cardíaca destina-se a uma assistência abrangente, não apenas pelo arsenal tecnológico de que se dispõe para a realização do cuidado de alta complexidade ${ }^{17,20,26}$, mas também deve garantir um adequado ambiente físico e controle da dimensão física, além de dispensar atenção às necessidades psicoemocionais do paciente, de forma a contribuir para sua recuperação ${ }^{17}$.

A disponibilidade do enfermeiro e sua equipe para estar ao lado do paciente e sua família, oferecendo suporte emocional e orientando-os, pode aliviar-lhes consideravelmente os anseios, o medo e as angústias causados pelo processo cirúrgico e hospitalização ${ }^{13,20}$.

Para realizar ações que promovam sentimentos positivos e formas educativas de amparo ao paciente, cabe ao enfermeiro, como responsável pela equipe de enfermagem, planejar uma assistência que o estimule a desafiar as situações que o afligem de modo a potencializar sua recuperação ${ }^{13}$.

Vale destacar que o enfermeiro é o profissional responsável pelo gerenciamento da unidade e pelas ações dos demais membros de sua equipe e, nesse sentido, pensamos o quanto é crucial o seu papel para que o trabalho de toda a equipe de enfermagem aconteça, apoiando-se na sistematização da assistência de enfermagem como ferramenta que favorece a organização do serviço.

A realização do processo de enfermagem é uma das atribuições do enfermeiro e consiste em cinco fases inter-relacionadas: histórico, diagnóstico, planejamento, implementação e avaliação. Diagnósticos de enfermagem foram identificados em pacientes no pós-operatório de cirurgia cardíaca tais como: Padrão Respiratório Ineficaz, Comunicação Verbal Prejudicada, Integridade da Pele Prejudicada, Perfusão Tissular Ineficaz, Mobilidade Física Prejudicada, Déficit no Autocuidado para Banho-Higiene, Dor Aguda, Risco de Infecção e outros ${ }^{16,27}$. Identificar diagnósticos de enfermagem contribuirá no planejamento e adequação dos cuidados de enfermagem necessários.

Estudos ${ }^{15-17,25,27,28}$ revelam a importância do processo de enfermagem na assistência prestada ao paciente, pois permite formular um plano de cuidados de acordo com as necessidades individuais.

Nesse contexto, o enfermeiro, bem como sua equipe, a partir do domínio técnico e científico, exerce funções de cuidado, controle e observação, considerando a complexidade da cirurgia, o que exige competências profissionais específicas. Após a avaliação do paciente, ocorre a formulação e execução de um plano de cuidados necessário e adequado para a recuperação do paciente.

\section{Gerenciando a dor}

Outra ação de enfermagem que está presente no cuidado dispendido ao paciente submetido a cirurgia cardiaca está diretamente relacionada a dor. A maioria dos pacientes após a cirurgia cardíaca sofre de dor moderada a grave. Barreiras individuais e atitudes dos pacientes em relatar a dor podem resultar em seu manejo inadequado. Assim, tornam-se necessárias intervenções preventivas e/ou educativas inovadoras para o alívio da dor pós-operatória.

A intensidade dolorosa mantém relações diretas com os sinais vitais apresentados pelo paciente, pois mudanças na frequência cardíaca, respiratória e na pressão arterial, principalmente, determinam a dimensão da dor e sua influência no pós-operatório. Destaca-se que a avaliação de pacientes que sentiram dor intensa e/ou insuportável antes da primeira renovação de curativos no pós-operatório teve a intensidade da dor reduzida mediante esse procedimento, enfatizando o benefício do cuidado de enfermagem na promoção do conforto do paciente ${ }^{29}$.

Com isso, a dor deve ser uma preocupação do enfermeiro e sua equipe, pois resulta no desenvolvimento de complicações e interfere diretamente no tempo de internação, nos padrões de sono, repouso, funções orgânicas, na dificuldade em restabelecer parâmetros vitais adequados, como capacidade respiratória, térmica e cardiocirculatória, em detrimento do agravante cirúrgico ${ }^{16,17,28,30}$. Nesse sentido, o enfermeiro, enquanto líder de sua equipe, desempenha papel fundamental na avaliação da dor e posterior implementação de estratégias e intervenções que poderão contribuir com a prática clínica.

A capacitação da equipe de enfermagem por meio da utilização de uma ferramenta como a escala visual numérica, para detecção da queixa álgica em pacientes no pós-operatório de cirurgia cardíaca, possibilita mensurar adequadamente a dor e escolher qual o melhor e mais seguro entre os diferentes tipos de tratamentos existentes. A utilização de uma escala oferece alívio efetivo à dor de acordo com o julgamento apropriado, possibilitando a humanização do cuidado na medida em que é valorizada a subjetividade e a satisfação das necessidades do paciente ${ }^{31}$.

Outro estudo descreve a validade, sensibilidade e especificidade de duas ferramentas de avaliação da dor amplamente utilizadas para crianças após cirurgias cardíacas, mostrando-se úteis na detecção de dor nessa população vulnerável ${ }^{32}$. Existe alta confiabilidade de escalas em capturar adequadamente a dor em pacientes em estado crítico não verbal, sendo os enfermeiros os profissionais da equipe de enfermagem capacitados a utilizar essas escalas de dor com facilidade e compreensão ${ }^{30}$.

A identificação da dor por meio do uso da Taxonomia II da NANDA-I em conjunto com o uso de instrumentos unidimensionais, como a escala de dor numérica, também são extremamente importantes para a identificação e descrição da dor, uma vez que a dor é referida como um fenômeno subjetivo e multimensional ${ }^{28}$.

Nesse sentido, a equipe de enfermagem deve ter conhecimento teórico-prático para a identificação e avaliação da dor após cirurgia cardíaca a fim de manter o conforto e acelerar a recuperação do paciente.

Ainda, deve-se destacar um estudo que mostrou enfermeiros de cuidados críticos utilizando diferentes combinações de sinais 
de dor, concluindo que o paciente em ventilação mecânica após a cirurgia cardíaca apresentou um "padrão de sugestão de prevenção primária da dor" evidenciado por alterações fisiológicas e um "padrão de sugestão de comportamento da dor" evidenciado por comportamentos, como, por exemplo, "careta" 33 .

No gerenciamento da dor, a equipe de enfermagem também deve atuar de forma precisa e eficaz no que diz respeito à infusão de drogas a fim de manter o conforto do paciente, bem como saber identificar a necessidade de complementação e manutenção de analgesia ${ }^{28}$, através da avaliação física, das queixas expostas pelo paciente e de comportamentos expressivos ${ }^{17,28}$.

Assim, com o intuito de auxiliar no afastamento da dor e do sofrimento, a manutenção do conforto merece atenção de toda a equipe de enfermagem, pois, além da implementação das terapias farmacológicas e não farmacológicas direcionadas à sintomatologia apresentada, requer a promoção de um ambiente terapêutico iluminado e silencioso ${ }^{17}$.

É possível influenciar o comportamento da dor, fornecendo suporte promissor, por meio de uma intervenção de enfermagem eletrônica para o alívio e manejo da dor pós-operatória. A intervenção incluiu uma sessão via internet de 30 minutos no pré-operatório e duas sessões de reforço no pós-operatório. Foram transmitidas mensagens eletrônicas por meio de vídeos, animações, histórias e textos. Os pacientes que receberam a intervenção relataram significativamente menos interferência da dor ao respirar e tossir, exibiram menos barreiras relacionadas a dor e consumiram mais medicação opioide do que o grupo controle; no entanto, não houve diferença na intensidade da dor entre os grupos ${ }^{34}$.

Pacientes no pós-operatório de cirurgia cardíaca foram convidados a ouvir música, de acordo com a preferência deles, apresentando-se como uma intervenção segura e eficaz em reduzir a dor. Fato que promove a autonomia da enfermagem e a capacidade de esta afetar o meio ambiente dos pacientes, além de ser uma terapia de baixo custo e sem efeitos colaterais ${ }^{35}$.

Além disso, o manejo da dor da criança realizado pelos profissionais de enfermagem, na perspectiva de familiares, pode estar relacionado à confiança na equipe, pelas ações desempenhadas e por atenderem prontamente os chamados das mães respondendo às suas dúvidas ${ }^{36}$.

O déficit na formação profissional de enfermeiros para o manejo da dor é realidade presente nos serviços de saúde ${ }^{13,36}$. Pode-se inferir que a capacitação em serviço necessita ser mantida como um programa de gestão, comprovando que a apropriação do conhecimento modifica comportamentos e qualifica a assistência ${ }^{31}$.

\section{A comunicação como ferramenta no cuidado de enfermagem}

A comunicação verbal constitui-se como um dos principais elementos dos cuidados de enfermagem e se apresenta como uma prioridade cada vez maior e mais complexa no processo cirúrgico. Haja vista, que proporciona a interação da equipe com o paciente e a família; a criação de um ambiente saudável, humanizado e sistematizado; além de esclarecer e orientar acerca das providências e atitudes após a cirurgia, contribuindo com a diminuição da ansiedade do paciente e um pós-operatório tranquilo ${ }^{13,17}$.

Existem algumas estratégias de comunicação que a equipe de enfermagem pode usar para amenizar o estresse do paciente, como: uso adequado das técnicas de comunicação verbal, captando-as e interpretando-as adequadamente; demonstrar confiança; e buscar criar vínculo com o paciente. Com isso, é possível que esses profissionais identifiquem suas necessidades de diálogo, escuta e orientações que melhorem o suporte ao paciente ${ }^{13}$.

A comunicação inadequada ou falta de informação da equipe com os familiares dos pacientes é considerada como fator de descuido ${ }^{36}$, evidenciando que a interação entre a família e a equipe de saúde constitui-se em um dos importantes esforços para o sucesso dessa prática.

Um fator importante que pode prejudicar a comunicação entre o paciente e a equipe de enfermagem é o uso da alta tecnologia. Esta tem sido considerada determinante no comportamento dos profissionais de enfermagem, no modo como cuidam e se relacionam com os pacientes $^{26}$. Assim, a presença e o uso de alta tecnologia podem afastar ou mesmo tornar o profissional de enfermagem indiferente às limitações do paciente. A equipe de enfermagem deve estar atenta para usar a tecnologia a seu favor, de modo que esta nunca deve se sobrepor à humanização do atendimento, necessária ao cuidado de qualidade.

$\mathrm{O}$ uso da comunicação verbal pela equipe de enfermagem, como ferramenta ou estratégia para o cuidado, demonstra-se eficaz em situações vivenciadas pelo paciente e seu familiar, além de contribuir para fundamentar uma assistência humanizada, com vista a atendê-lo em suas especificidades e promover maior conforto na sua recuperação ${ }^{13}$.

Dessa forma, a equipe de enfermagem deve encorajar a verbalização do paciente, ouvi-lo, reconhecer e respeitar seus sentimentos, percepções e emoções. A interação com o paciente estabelece uma relação de confiança e tranquilidade, desempenhando papel fundamental para a qualidade da assistência prestada.

Dessa forma conclui-se que o paciente submetido à cirurgia cardíaca demanda da equipe de saúde visão ampla acerca das suas necessidades fisiológicas e/ou psicossociais, com intuito de promover um cuidado integral, individualizado e humanizado.

Os resultados deste estudo mostram um cuidado de enfermagem especializado, que deve ser planejado de forma individualizada, e a necessidade de os profissionais terem conhecimentos científicos e técnicos para sua atuação nesse contexto. Além disso, cuidados referentes à manutenção do débito cardíaco, da integridade tecidual, do equilíbrio hidroeletrolítico e da oxigenação são desenvolvidos pela equipe de enfermagem.

Cabe ainda a essa equipe assistir e acolher esse paciente emocionalmente, ação esta necessária para desenvolver de fato uma assistência humanizada.

O enfermeiro como líder da equipe tem o papel de gerenciar a unidade, planejar os cuidados por meio da sistematização da assistência de enfermagem e capacitar sua equipe para atuarem 
juntos na recuperação desse paciente. O enfermeiro, ao utilizar o processo de enfermagem como ferramenta para a organização do cuidado, bem como a comunicação eficaz, contribui potencialmente para a segurança e diminuição de possíveis traumas cirúrgicos desses pacientes.

Assim, competências devem ser desenvolvidas continuamente aos profissionais da equipe de enfermagem a fim de implementar ações de cuidado eficazes na prática diária de pacientes submetidos a cirurgia cardíaca.
Os gestores das instituições têm o papel de desenvolver estratégias que promovam a capacitação de seus profissionais, bem como os centros formadores devem ter a preocupação de preparar trabalhadores aptos para atuar nesse cenário.

Novos estudos devem ser realizados no sentido de identificar as competências necessárias à equipe de enfermagem, bem como as estratégias institucionais realizadas para a capacitação da equipe nesse contexto, pois é incontestável o seu papel na recuperação do paciente.

\section{REFERÊNCIAS}

1. Ferreira JBB. O processo de descentralização e regionalização da saúde no estado de São Paulo. In: Ibañez N, Elias PEM, Seixas PHD, organizadores. Política e Gestão Pública em Saúde. São Paulo: Hucitec; 2011. p. 731-58

2. Organização Pan-Americana da Saúde. Organização Mundial da Saúde. Saúde nas Américas. Vol. 1. Washington: OPAS; 2007.

3. Malta DC. Doenças crônicas não transmissíveis, um grande desafio da sociedade contemporânea. Ciênc Saúde Coletiva. 2014;19(1):4. http://dx.doi.org/10.1590/1413-81232014191.0084

4. World Health Organization [Internet]. The 10 leading causes of death in the world, 2000 and 2012. Disponivel em: http://www.who. int/mediacentre/factsheets/fs310/en/. Acesso em: 23 jul. 2014.

5. Sala A. As doenças do aparelho circulatório no Estado de São Paulo. Bepa. 2011;8(88):1-8.

6. Mendes JDV, Bittar OJNV. Saúde pública no estado de São Paulo: informações com implicações no planejamento de programas e serviços. Rev Adm Saúde. 2010;Ed. Especial (Suppl):5-71.

7. Pinto Junior VC, Fraga MNO, Freitas SM. Análise das portarias que regulamentam a Política Nacional de Atenção Cardiovascular de Alta Complexidade. Rev Bras Cir Cardiovasc. 2012;27(3):463-68. http://dx.doi.org/10.5935/1678-9741.20120077

8. Galdeano LE, Rossi LA, Santos CB, Dantas RAS. Diagnósticos de enfermagem no perioperatório de cirurgia cardíaca. Rev Esc Enferm USP. 2006;40(1):26-33.

http://dx.doi.org/10.1590/S0080-62342006000100004

9. Laizo A, Delgado FEF, Rocha GM. Complicações que aumentam o tempo de permanência na unidade de terapia intensiva na cirurgia cardíaca. Rev Bras Cir Cardiovasc. 2010;25(2):166-71. http://dx.doi.org/10.1590/S0102-76382010000200007

10. Rocha LA, Maia TF, Silva LF. Diagnósticos de enfermagem em pacientes submetidos à cirurgia cardíaca. Rev Bras Enferm. 2006;59(3):321-6. http://dx.doi.org/10.1590/S0034-71672006000300013

11. Atik FA, Garcia MFMA, Santos LM, Chaves RB, Faber CN, Corso $\mathrm{RB}$, et al. Resultados da implementação de modelo organizacional de um serviço de cirurgia cardiovascular. Rev Bras Cir Cardiovasc. 2009;24(2):116-25.

http://dx.doi.org/10.1590/S0102-76382009000200005

12. Rodrigues CG, Senger R, Guido LA, Linch GFC. Cardiac surgery postperative: diagnosis and nursing interventions. J Nursing UFPE. 2010:4(1):391-400.

http://dx.doi.org/10.5205/01012007
13. Gonçalves RMDA, Pereira MER, Pedrosa LAK, Silva QCG, Abreu RMD. A comunicação verbal enfermeiro-paciente no perioperatório de cirurgia cardíaca. Ciênc Cuid Saúde. 2011;10(1):27-34. http://dx.doi.org/10.4025/cienccuidsaude.v10i1.8681

14. Gois CFL, Dantas RAS. Estressores em uma unidade pósoperatória de cirurgia torácica: avaliação da enfermagem. Rev Latino-Am Enfermagem. 2004;12(1):22-7. http://dx.doi.org/10.1590/S0104-11692004000100004

15. Lira ALBC, Araújo WM, Souza NTC, Frazão CMFQ, Medeiros ABA. Mapeamento dos cuidados de enfermagem para pacientes em pós-operatório de cirurgia cardíaca. Rev Rene. 2012;13(5):1171-81.

16. Pivoto FL, Lunardi-Filho WD, Santos SSC, Almeida MA, Silveira RS. Diagnósticos de enfermagem em pacientes no período pós-operatório de cirurgias cardíacas. Acta Paul Enferm. 2010;23(5):665-70.

17. Duarte SCM, Stipp MAC, Mesquita MGR, Silva MM. O cuidado de enfermagem no pós-operatório de cirurgia cardíaca: um estudo de caso. Esc Anna Nery. 2012;16(4):657-65. http://dx.doi.org/10.1590/S1414-81452012000400003

18. Souza MT, Silva MD, Carvalho R. Revisão integrativa: o que é e como fazer. Eisntein. 2010;8(1 Pt 1):102-6.

19. Melnyk BM, Fineout-Overholt E. Evidence-based practice in nursing \& healthcare: a guide to best practice. 2nd edition. Philadelphia: Lippincott Williams \& Wilkins; 2005. p. 3-24.

20. Melo HC, Araújo SEG, Santos VEFA, Veríssimo AVR, Alves ERP, Souza MHN. O ser-enfermeiro em face do cuidado à criança no pós-operatório imediato de cirurgia cardíaca. Esc Anna Nery. 2012;16(3):473-9

http://dx.doi.org/10.1590/S1414-81452012000300007

21. Starks B, Harbert C. Aspiration prevention protocol: decreasing postoperative pneumonia in heart surgery patients. Crit Care Nurse. 2011;31(5):38-45.

http://dx.doi.org/10.4037/ccn2011462

22. Brindle CT, Wegelin JA. Prophylactic dressing application to reduce pressure ulcer formation in cardiac surgery patients. $J$ Wound Ostomy Continence Nurs. 2012;39(2):133-42. http://dx.doi.org/10.1097/WON.0b013e318247cb82

23. Nilsson U. Soothing music can increase oxytocin levels during bed rest after open-heart surgery: a randomised control trial. J Clin Nurs. 2009;18(15):2153-61. http://dx.doi.org/10.1111/j.1365-2702.2008.02718.x 
24. Albert NM, Gillinov AM, Lytle BW, Feng J, Cwynar R, Blackstone EH. A randomized trial of massage therapy after heart surgery. Heart Lung. 2009;38(6):480-90. http://dx.doi.org/10.1016/j.hrtlng.2009.03.001

25. Magalhães MGPA, Alves LMO, Alcantara LFM, Bezerra SMMS. Mediastinite pós-cirúrgica em um hospital cardiológico de Recife: contribuições para a assistência de enfermagem. Rev Esc Enferm USP. 2012;46(4):865-71.

http://dx.doi.org/10.1590/S0080-62342012000400012

26. Silva RCL, Kaczmarkiewicz CC, Cunha JJSA, Meira IC, Figueiredo NMA, Porto IS. O significado da tecnologia na assistência de enfermagem em pós-operatório imediato de cirurgia cardíaca. Rev SOCERJ. 2009;22(4):210-18.

27. Cruz APO, Lopes R. Diagnóstico de enfermagem no pósoperatório de cirurgias cardíacas. Salusvita. 2010;29(3):293-312.

28. Faria-Filho GS, Caixeta LR, Stival MM, Lima LR. Dor aguda: julgamento clínico de enfermagem no pós-operatório de cirurgia cardíaca. Rev Min Enferm. 2012;16(3):400-9

29. Miranda AFA, Silva LF, Caetano JA, Sousa AC, Almeida PC. Avaliação da intensidade de dor e sinais vitais no pós-operatório de cirurgia cardíaca. Rev Esc Enferm USP. 2011;45(2):327-33. http://dx.doi.org/10.1590/S0080-62342011000200004

30. Marmo L, Fowler S. Pain assessment tool in the critically ill post-open heart surgery patient population. Pain Manag Nurs. 2010;11(3):134-40.

http://dx.doi.org/10.1016/j.pmn.2009.05.007
31. Keller C, Paixão A, Moraes MA, Rabelo ER, Goldmeier S. Escala da dor: implantação para pacientes em pós-operatório imediato de cirurgia cardíaca. Rev Esc Enferm USP. 2013:47(3):621-5. http://dx.doi.org/10.1590/S0080-623420130000300014

32. Bai J, Hsu L, Tang Y, van Dijk M. Validation of the COMFORT Behavior scale and the FLACC scale for pain assessment in Chinese children after cardiac surgery. Pain Manag Nurs. 2012;13(1):18-26.

http://dx.doi.org/10.1016/j.pmn.2010.07.002

33. Connor LO. Critical care nurses' judgement of pain status: a case study design. Intensive Crit Care Nurs. 2012;28(4):215-23. http://dx.doi.org/10.1016/j.iccn.2012.01.004

34. Martorella G, Côté J, Racine M, Choinière M. Web-based nursing intervention for self-management of pain after cardiac surgery: pilot randomized controlled trial. J Med Internet Res. 2012:14(6):e177

http://dx.doi.org/10.2196/jmir.2070

35. Özer N, Karaman Özlü Z, Arslan S, Günes N. Effect of music on postoperative pain and physiologic parameters of patients after open heart surgery. Pain Manag Nurs. 2013;14(1):20-8. http://dx.doi.org/10.1016/j.pmn.2010.05.002

36. Nascimento LC, Strabelli BS, Almeida FCQG, Rossato LM Leite AM, Lima RAG. O manejo da dor em crianças, no pósoperatório tardio de cirurgia cardíaca, pelos profissionais de enfermagem, na ótica das mães. Rev Latino-Am Enfermagem. 2010;18(4):709-15

http://dx.doi.org/10.1590/S0104-11692010000400008 